\title{
Computer simulation of the influence of thermal conditions on the performance of conventional and unconventional lithium-ion battery geometries
}

\author{
D. Miranda ${ }^{1,2}$, C. M. Costa ${ }^{1,3^{*}}$, A. M. Almeida ${ }^{1}$, S. Lanceros-Méndez ${ }^{4,5}$ \\ ${ }^{1}$ Centro de Física, Universidade do Minho, 4710-057 Braga, Portugal \\ ${ }^{2}$ Instituto Politécnico do Cávado e do Ave, Campus do IPCA, 4750-810 Barcelos, \\ Portugal \\ ${ }^{3}$ Centro de Química, Universidade do Minho, 4710-057 Braga, Portugal \\ ${ }^{4}$ BCMaterials, Basque Center Centre for Materials, Applications and Nanostructures, \\ UPV/EHU Science Park, 48940 Leioa, Spain \\ ${ }^{5}$ IKERBASQUE, Basque Foundation for Science, 48013 Bilbao, Spain
}

\begin{abstract}
:
Thermal analysis is a fundamental issue for the proper evaluation of the performance of lithium ion batteries. Thus, this work reports on the theoretical simulation of the effect of different thermal conditions on the performance of batteries with conventional and nonconventional geometries.

The investigated geometries include conventional geometries (layer by layer), interdigitated, horseshoe, spiral, ring, antenna and gear that can be fabricated by printed technologies for flexible / wearable electronic device applications. The thermal conditions are isothermal, adiabatic and environmental conditions and the computational simulations are based on the electrochemical (Newman/Doyle/Fuller) model.

Generally, the best battery performance is obtained for interdigitated and gear geometries, regardless of thermal conditions. The dissipated ohmic heat is mainly influenced by the maximum distance that the ions move until their intercalation and the thickness of the separator. The present work allows the proper design of the batteries in order to optimize performance for specific applications.
\end{abstract}

Keywords: lithium-ion batteries; battery geometry; thermal conditions; computer simulations 


\section{Introduction}

Electrical energy is increasingly being obtained through renewable sources, such as, solar, wind, waves, bioenergy and geothermal, leading to the need for efficient energy storage systems $[1,2]$.

These energy storage systems are essential for portable electronic devices such as mobile phone and computers but also for transportation systems, i.e, hybrid electric vehicles (HEVs) [3] and pure electric vehicles (PEVs) [4], in which lithium-ion batteries are the most commonly used energy storage systems [5].

Lithium-ion batteries are light weight, show high energy density $\left(210 \mathrm{Wh}_{\mathrm{kg}}{ }^{-1}\right)$, low charge loss, no memory effect, large service-life and high number of charge/discharge cycles [6].

The main constituents of a lithium-ion battery are the anode (negative electrode), the cathode (positive electrode) and the separator/electrolyte and the main issues to improve its performance are specific energy, power, safety and reliability [7].

Lithium-ion batteries are extremely sensitive to certain temperature ranges, typically being functional between $-20^{\circ} \mathrm{C}$ and $\sim 50$ to $60^{\circ} \mathrm{C}$ [8]. During the cycling of a battery, its temperature increases and, if the temperature exceeds certain limits, exothermic reactions can occur, resulting in increased internal pressure, rupture or even explosion of the battery $[9,10]$.

For certain applications, in which high discharge rates are required, the thermal management of the batteries is critical in order to optimize performance and to avoid structural damage and consequently loss battery performance during operation $[2,10,11]$. Thermal management systems (TMS) are implemented with the objective to avoid overheating of battery packs [12]. Most TMS make use of cooling mediated by air [13], liquid [14], heat pipe [15], or phase change materials (PCM) [8]. Recently, the cooling efficiency of the parallel air-cooled battery thermal management system (BTMS) was optimized with U-type flow. For the process at 5C discharge rate, the temperature difference among the battery cells is reduced by $70 \%$ after optimization, leading to a power consumption reduction of $32 \%$ [16]. Further, the thermal behavior of batteries was analyzed in thermal/climate chambers which the use forced air convection to distribute heat, introducing a novel thermal management system which maintains cell temperature reliably whilst minimizing also the thermal gradients. The proposed method reduces 
external temperature deviations of the cell to within $1.5^{\circ} \mathrm{C}$, providing reliable data for parameterizing a thermally discretized model [17].

The thermal performance of a Li-ion battery for an phase change material (PCM) loaded with carbon fibers was studied, showing that the carbon fibers have a significant influence in the thermo-physical properties of the PCM through the improvement of the effective thermal conductivity [18]. Further, the thermal conductivity of the PCM with double copper mesh increases by $36 \%$ compared with that of the PCM composed of paraffin (PA) and expanded graphite (EG) [19].

The analysis of the thermal conditions is very important in order to optimize those systems and for batteries applied into microgrids structures [20-23].

The thermal performance of battery modules under different cell arrangement structures was investigated by three-dimensional computational fluid dynamics (CFD) method and it was verified that when the fan is located on top of the module, the best cooling performance is achieved and the most desired structure with forced air cooling is the cubic arrangement [24].

Finally, the thermal behavior of high-power lithium-ion cells was investigated using an accelerating rate calorimeter. It is shown that heat generation is less marked at higher Crates, resulting in a larger irreversible heat component during charging and discharging at high current rates [25].

The influence of the thermal conditions on the performance of lithium ion battery should be analyzed for each battery component [26]. In particular, the thickness of the electrodes strongly affects the overall heat generation [27], as the generated ohmic heat is larger for thicker electrodes [27].

The size of each active material also affects the generation of heat [11] and the effect of particle size for the $\mathrm{LiMn}_{2} \mathrm{O}_{4}$ active material was studied by using a thermal model [28], demonstrating the higher generation of heat for larger particles size [28]. Further, the geometry of the battery also influences its thermal behavior [10, 29]. The thermal model for the behavior of $\mathrm{LiNi}_{\mathrm{x}} \mathrm{Co}_{\mathrm{y}} \mathrm{Mn}_{\mathrm{z}} \mathrm{O}_{2}$ (NCM) lithium-ion batteries for high charge/discharge processes (up to $8 \mathrm{C}$ ) compares well with experimental, allowing to assess the influence of the external heat release conditions and charge/discharge rate on the thermal behavior of the batteries. It is concluded that favorable heat release conditions or effective and active thermal management are the key for the thermal control of lithiumion batteries [30]. 
The thermal behavior of batteries with cylindrical, prismatic and pouch cell

geometries was analyzed under different electrical loads and cooling conditions [31].

In relation to cylindrical cell geometries, it is observed a decreasing heat transfer resistance with increasing radius due to adiabatic condition at the cell core. On the other hand, differential temperature across the cell thickness must be considered for prismatic cells [31].

The thermal behavior of a lithium ion battery during galvanostatic discharge was analyzed by computer simulation showing that higher cell temperatures raise the risk of thermal runaway (TR) and more rapid degradation of the cell [32].

A thermal runaway (TR) propagation model was applied to a large format lithium ion battery module and it was concluded that TR propagation can be prevented by increasing the TR triggering temperature by modifying the separator, reducing the total electric energy released during TR by discharging the battery and enhancing the heat dissipation by increasing the convection coefficient [33]. The effect of heat sources in the thermal runaway processes of lithium-ion batteries composed of different chemistries using accelerating rate calorimetry (ARC) and differential scanning calorimetry (DSC) was analyzed, showing that internal short circuit is not the only way to thermal runaway, but can also lead to extra electrical heat, which is comparable with the heat released by the chemical reactions [34].

New lithium-ion unconventional battery geometries, such as ring, spiral, horseshoe, antenna and gear have been reported in [29], as they can be fabricated by 3D printing technologies and provide more adequate geometries for specific applications.

Considering the relevance of the thermal issues in lithium-ion batteries, the novelty of this work is to evaluate and analyze the effect of different thermal conditions on those new unconventional geometries (interdigitated, horseshoe, spiral, ring, antenna and gear) as well as in conventional geometries, for comparison. The applied scan rates are correlated to the area of each component and the battery geometries used are based on graphite and lithium iron phosphate $\left(\mathrm{LiFePO}_{4}\right)$ as active material for the anode and cathode electrode, respectively.

These geometries are proposed as an alternative for portable and wearable devices considering their better integration into devices and can be produced by 3D printing 
techniques with industrial scalability. These geometries were recently reported [29], but their thermal characteristics have not been evaluated yet. In this work, the thermal properties were analyzed as a function of the maximum distance for the ions to move to the current collector, d_max, distance between current collectors, d_cc, and the thickness of the separator and electrodes. Thus, this work will allow the proper dimensional design and thermal condition optimization of those batteries. 


\section{Experimental section}

\subsection{Materials and preparation of the electrodes}

For the validation of the theoretical thermal model, a full-cell was developed.

For the preparation of the electrodes (anode and cathode), carbon coated lithium iron phosphate, $\mathrm{C}-\mathrm{LiFePO}_{4}$ (LFP, Particle size: D10 $=0.2 \mu \mathrm{m}, \mathrm{D} 50=0.5 \mu \mathrm{m}$ and D90=1.9 $\mu \mathrm{m}$ ), poly(vinylidene fluoride) (PVDF, Solef 5130) and N,N'-dimethyl propylene urea (DMPU) were acquired from Phostech Lithium, Solvay and LaborSpirit, respectively. Timrex SLG3 graphite particles and carbon black (Super P-C45) were obtained from Timcal Graphite \& Carbon.

The electrodes were prepared by mixing LFP (for the cathode) or graphite (for the anode) as active materials, Super P (conductive additive), and PVDF (polymer binder) in DMPU solvent with a weight ratio of 80:10:10 (wt.\%), i.e, $1 \mathrm{~g}$ of solid material for $2.25 \mathrm{~mL}$ of DMPU. The electrodes were dried at $90^{\circ} \mathrm{C}$ in vacuum over night before being transferred into the glove-box [35].

\subsection{Full-cell manufacturing and testing}

Swagelok type cells were assembled in a home-made argon-filled glove box: the graphite based electrodes ( $8 \mathrm{~mm}$ diameter) were used as anode material; glass microfiber separators (Whatman grade GF/A) (10 mm diameter) were used as separators; $1 \mathrm{M} \mathrm{LiPF}_{6}$ in ethylene carbonate-diethyl carbonate (EC-DEC, 1:1 vol) (Solvionic) was used as electrolyte and LFP based electrodes were used as cathode material ( $8 \mathrm{~mm}$ diameter).

The prelithiation of the graphite electrodes was previously achieved by placing them in direct contact with an electrolyte-wetted Li foil for 2 hours, under slight pressure. The active mass loading of the anode and cathode used in the full cell were $\sim 1.20$ and 2.92 mg.cm ${ }^{-2}$, respectively.

The full battery was cycled at $25^{\circ} \mathrm{C}$ from $2.5 \mathrm{~V}$ to $3.8 \mathrm{~V}$ at $\mathrm{C} / 10$ rate $\left(\mathrm{C}=170 \mathrm{~mA} . \mathrm{g}^{-1}\right)$ using a Landt CT2001A Instrument. 


\section{Theoretical model: parameters, initial values and boundary conditions}

\subsection{Theoretical simulation model}

Simulations were performed by applying the electrochemical model based on the Newman/Doyle/Fuller equations coupled with the thermal behaviour.

This electrochemical model describes the electrochemical processes that occurs in battery components, electrodes, separator and current collectors, including the thermal behaviour.

The flowchart of the coupling of both models is present in figure 1.

Figure 1 - Flowchart of the proposed method.

Thus, the initial temperature is defined and the battery operates in the discharge process, allowing to determine the heat produced in the electrodes through reversible, reaction and ohmic heats, and in the separator, just by ohmic heat. These produced heat is the output of the electrochemical model and the input of the thermal model, in which conduction and absorption heat along the battery components is determined as well as the temperature reached by the battery.

The simulations were carried out by implementing the finite element method for different 2D battery geometries in a typical lithium-ion battery structure: [porous negative electrode, $\left(\mathrm{Li}_{\mathrm{x}} \mathrm{C}_{6}\right) \mid$ porous membrane of glass micro fiber soaked in $1 \mathrm{M}$ lithium hexafluorophosphate $\left(\mathrm{LiPF}_{6}\right)$ in ethylene carbonate-diethyl carbonate (EC-DEC) | porous positive electrode, $\left.\left(\mathrm{Li}_{\mathrm{x}} \mathrm{FePO}_{4}\right)\right]$.

Figure 2 represents the conversion between 3D and 2D conventional geometry for the application of the model and the corresponding equations.

Figure 2 - Schematic representation of the conversion between a 3D to a 2D conventional geometry for the application of the model and the corresponding equations. 
As illustrated in figure 2, the electrode volume is related to the amount of lithium ions present in the electrode (cathode) in the $3 \mathrm{D}$ representation. In the case of the $2 \mathrm{D}$ representation, the amount of lithium ions is related to the area of the electrode, which is set constant for all investigated geometries.

In this work, the applied scan rates are correlated with the area of each component, the cathode electrode area being set to $8 \times 10^{-8} \mathrm{~m}^{2}$.

The finite element calculations describing the electrochemical and thermal models were carried out by programming MATLAB scripts in order to solve the equations describing the behavior of the different battery constituents (electrodes and separator) in an ideal cell without solid electrolyte interface (SEI) formation, as presented in Table 1. The size of the mesh was refined according to the dimensions of the different geometries of the battery. The degree of porosity of the electrodes, defined as the space between the particles of active electrode material, is shown in table 2 for both electrodes.

In all batteries, a current density of $17.5 \mathrm{~A} / \mathrm{m}^{2}$ is applied at the discharge rate $i_{\text {app. }}$ Thus, it is possible to reach a maximum discharge rate of $500 \mathrm{i}_{\text {app }}$, since the area of the current collectors in all batteries is in the order of the $\mu \mathrm{m}^{2}$.

The impedance was measured for each geometry at frequencies ranging from $10 \mathrm{mHz}$ to $1 \mathrm{MHz}$ with a potential perturbation amplitude of $0.01 \mathrm{~V}$ and with the following parameters: film resistance of the positive electrode: $0.0065 \mathrm{~m}^{2} \cdot \mathrm{S}^{-1}$; film resistance of the negative electrode: $1 \times 10^{-5} \mathrm{~m}^{2} \cdot \mathrm{S}^{-1}$; double layer capacitance of the positive electrode: 0.2 F. $\mathrm{m}^{-2}$; double layer capacitance of the negative electrode: $0.2 \mathrm{~F}^{-\mathrm{m}^{-2}}$; current collector resistance at each current collector: $1.1 \times 10^{-4} \mathrm{~m}^{2} \cdot \mathrm{S}^{-1}[36]$.

Table 1 shows the equations governing the different constituents (cathode, anode and electrolyte/separator) in the operation of a lithium-ion battery as well as the corresponding thermal behavior. The equations describing the different phenomena occurring in the electrodes and electrolyte/separator include: the diffusion and ionic conductivity of lithium ions in the electrolyte and electrodes, the open circuit voltage and the mass transport flux, the relation between the potential of the electrolyte and the local current density on the electrodes (Ohm's law), the diffusion of lithium ions in the active material and the kinetics of the heterogeneous reaction at the electrode/electrolyte interface.

Table 1 - Equations governing the main phenomena of a battery [32, 37]. The identification of each symbol is provided in the nomenclature section. 


\subsection{Specific parameters and initial values}

The values of the parameters used for the different components of each battery geometry are listed in Table 2 [38, 39]. The areas of all components were maintained constant in the computer simulations as shown in [40].

Table 2 - Values of the parameter values used in the simulations. The nomenclature is shown in Annex I.

Figure 3 shows the schematic representation of each of the evaluated geometries, conventional, interdigitated, gear, horseshoe, spiral, antenna and ring.

The different geometries are designed to improve integration in different applications and the batteries can be fabricated by printing technologies. For example, the ring and gear geometries can be applied in watches, mobile phones, medical devices and spiral and antenna geometries are suitable for smart and gift cards, smart toys, sensors and actuators, medical devices (e.g. transdermal drug delivery (TDD) systems.

Figure 3 - Schematic representation of the different battery geometries

Table 3 - Values of the dimensional parameters for each battery design. The nomenclature for each parameter is provided in the nomenclature section.

Table 3 shows the values of the dimensions used for the simulation of the different batteries such as distance between collectors and thickness of the electrodes and separator, among others. The parameter d_max is particularly relevant for analyzing battery performance as it represents the maximum distance of the ions to the collectors. The number of digits in the interdigitated and gear geometries is eight considering the area of the electrodes. The number of digits allows to increase the contact area between the different battery components. 


\subsection{Boundary conditions}

The boundary conditions were defined accordingly to the electrochemical (Newman/Doyle/Fuller) and thermal models. The boundary conditions are schematically presented in Figure 4 and defined in Table 4.

As the boundary conditions are the same for all geometries, just the ones for conventional geometry will be presented, as an example. Table 4 shows these boundary conditions addressing the schematic representation of the conventional geometry, illustrated in Figure 4.

In Table 4 and Figure 4, the boundary conditions are identified from 1 to 7.

According to Figure 4, the boundary condition indicates that there is no ion flux. Regarding the thermal model in the adiabatic condition, there is no heat transfer with the external environment, as defined by boundary condition 1. Also, at adiabatic condition the external temperature is not applicable (boundary condition 6). In contrast, for the thermal model with different conditions (cold, regular and hot temperatures) there is heat transfer with the external environment (boundary condition 1) and an external temperature was defined according to the applied thermal condition (boundary condition $6)$.

For the interfaces between the electrodes and the separator, as well as between the electrodes and the current collectors/external medium, the boundary conditions 2, 3, 4, 5 were defined. These boundary conditions define the value of the ionic conductivity/diffusion, concentration of lithium ions and electric conductivity for both sides of the interface border.

Finally, the boundary condition 7 defines the values of the ionic diffusion and concentration of the lithium salt along the radius of the spherical particles of active material.

Table 4 - Summary of the boundary conditions implemented in the conventional geometry. 
Figure 4 - Schematic representation of the boundary conditions applied in the conventional geometry.

\section{Results and discussion}

Theoretical model simulations were thus applied in all different lithium-ion battery geometries in different thermal conditions: isothermal, adiabatic and environmental conditions (cold, regular and hot temperatures) keeping constant the area of the components.

The theoretical model was first validated with the experimental results obtained for the developed full cell.

The main objective is to evaluate how the performance of the batteries with different geometries are affected by the thermal conditions. The capacity value is in the form of ampere-hour per square meter $\left(\mathrm{Ah} \cdot \mathrm{m}^{-2}\right)$ - capacity per unit area- and depends on the electrode area as the area of the different components of the different geometries are kept constant.

\section{1. $\mathrm{LiC}_{6} / \mathrm{LiFePO}_{4}$ full-cell: Validation of the theoretical model}

The simulation model was validated by comparing the theoretical (with and without thermal model) and experimental results obtained for the $\mathrm{LiC}_{6} / \mathrm{LiFePO}_{4}$ full-cell with conventional geometry (figure 5).

Figure 5 shows experimental and simulation curves (with and without thermal model) for the full-cell at $298 \mathrm{~K}$ and at scan rate of $0.51 \mathrm{~A} \cdot \mathrm{m}^{-2}$.

Figure 5 - Voltage as a function of the delivered capacity at $0.51 \mathrm{~A}^{-2}$ for the $\mathrm{LiC}_{6} / \mathrm{LiFePO}_{4}$ full-cell with a conventional geometry.

Figure 5 shows a good agreement between experimental and theoretical (with thermal model) results. There is a slight deviations of the capacity of the real full-cell relative to the theoretical model below $3.2 \mathrm{~V}$, attributed to corresponding differences in the electronic conductivity values and also to the exact temperature value during the discharge process [41]. 
Thus, the good theoretical approximation allows the validation of the theoretical model with thermal conditions as evidenced by the observed differences between the theoretical model with and without thermal considerations.

\subsection{Battery performance of the various battery geometries at different thermal conditions}

Theoretical model simulations with thermal conditions were carried out for all geometries in different thermal conditions (isothermal, adiabatic and environmental: cold, regular and hot temperatures) varying scan rate between $1 i_{\text {app }}$ to $500 i_{\text {app }}$.

\subsubsection{Isothermal condition}

All geometries were tested at scan rates between $\mathrm{l}_{\text {app }}$ to $500 \mathrm{i}_{\text {app }}$ for a constant temperature of $298 \mathrm{~K}$, i.e, without applying the thermal equations.

A similar study has been already presented for all these geometries with lithium manganese oxide ( $\left.\mathrm{LiMn}_{2} \mathrm{O}_{4}, \mathrm{LMO}\right)$ as active material [29], which leads to differences based on specific electric and ionic conductivity values and lithium diffusion coefficients, among others, of the active materials [42].

Thus, the present investigations also allow to evaluate the influence of active material on battery performance.

Figure 6 shows the discharge capacity value as a function of the scan rate for all geometries under isothermal condition.

Figure 6 - Delivered capacity as a function of scan rate for all geometries under isothermal condition.

For low scan rates, no significant differences arise and all geometries show high capacity. At medium and high scan rates, the discharge capacity of the geometries follows this order: interdigitated, gear, horseshoe, spiral, antenna, ring and conventional.

It can be observed that the conventional geometry operates just up to $300 \mathrm{i}_{\text {app }}$ and for this scan rate, its discharge capacity value is $3.61 \mathrm{Ah} \cdot \mathrm{m}^{-2}$. It is also observed that all other geometries may operate at higher rates up to $500 \mathrm{i}_{\mathrm{app}}$. 
The interdigitated geometry shows the best performance for all scan rates. At $300 \mathrm{i}_{\text {app }}$, its capacity is 356 Ah.m ${ }^{-2}$. which is 98 times higher than the one for the conventional geometry.

The gear geometry closely follows the interdigitated one and at $300 \mathrm{i}_{\text {app }}$, the capacity value is $354 \mathrm{Ah} \cdot \mathrm{m}^{-2}$.

The different discharge capacity values observed for the geometries is ascribed to different internal resistance values, variations of the maximum distance, and distance between current collectors in the different geometries, as well as to variations of the dimensions of the components (electrodes and separator), as shown in table 3.

Thus, the main reason for the conventional geometry not operating at scan rates above $300 \mathrm{i}_{\text {app }}$ is due to the high thickness of the electrodes and separator in comparison to the other geometries, which limits the diffusion of ions.

The interdigitated and gear geometries show lower maximum distance values (d_max) and higher contact surface area between the electrodes than any other geometry, as can be seen in table 3 .

During the discharge process, the geometry effect is more significant for higher scan rates once it is required elevated mobility of ions and electrons.

By comparison with the literature [29], it is observed that the results with $\mathrm{LiFePO}_{4}$ or $\mathrm{LiMn}_{2} \mathrm{O}_{4}$ as active material are similar.

\subsubsection{Adiabatic condition}

All geometries were tested under adiabatic condition with an initial temperature of 298.15 $\mathrm{K}$ before the discharge process for all geometries. Figure $7 \mathrm{a}$ ) and $\mathrm{b}$ ) show the discharge capacity value and the temperature for all geometries at scan rate between $1 i_{\text {app }}$ and $500 \mathrm{i}_{\text {app }}$, respectively.

Figure 7 - Delivered capacity (a) and temperature (b) as a function of the scan rate for all geometries under adiabatic condition.

The discharge capacity value decreases when increasing the scan rate as it is shown in figure $7 \mathrm{a})$. Up to $200 \mathrm{i}_{\mathrm{app}}$, the discharge capacity value is practically the same for all 
geometries, being the differences observed in the discharge capacity value after $300 \mathrm{i}_{\text {app }}$ for the different geometries attributed to variation in the internal resistance of the batteries due to geometrical effects, as previously indicated.

The discharge capacity value is higher under adiabatic conditions when compared to isothermal condition for the same scan rates. The reason for this effect is due that heat produced for each geometry will be internally absorbed, leading to a temperature increase (figure $7 b$ ), which in turn affects the diffusion and ionic conductivity values (Table 1).

The conventional geometry only operates up to $300 \mathrm{i}_{\text {app }}$ and its discharge capacity value is $367.05 \mathrm{Ah} / \mathrm{m}^{2}$. For this geometry, the discharge value in the adiabatic condition is higher relative to the isothermal condition $\left(3.61 \mathrm{Ah} / \mathrm{m}^{2}\right)$, due to the increase of temperature and the corresponding effect on the diffusion and ionic conductivity values [43].

The conventional geometry has higher internal resistance due to longer distance between current collectors and larger thickness of the separator. Thus, it is observed that the higher discharge capacity caused by the increase of the temperature is not sufficient to overcome the losses associated to the high internal resistance.

Figure $7 b$ ) shows that the battery temperature increases with the scan rate up to $300 \mathrm{i}_{\text {app }}$ for all geometries, due to the heat produced by ohmic losses [44]. For scan rates above $300 \mathrm{i}_{\text {app }}$ it is observed that the temperature decreases as the scan rate increase, as the heat produced is not totally absorbed during the discharge cycle due to the low discharge time. Figure 7b) also shows that the interdigitated and gear geometries present lower temperature values relatively to the other geometries. The main reason for this behavior is due to the smaller separator thickness, lower maximum distances that ions cross until their intercalation (d_max) and higher contact surface between the electrodes.

In adiabatic conditions, the interdigitated geometry shows higher discharge capacity value for all scan rates in comparison to the other geometries, including the conventional geometry. As for the temperature value, the conventional geometry has higher value when compared to other geometries due to its higher internal resistance.

To evaluate the internal resistance of the battery, impedance measurements were carried out for the conventional and interdigitated geometries at $298.15 \mathrm{~K}$.

Figure 8 shows the Nyquist plot for both geometries in the adiabatic condition at frequencies between $1 \mathrm{MHz}$ to $0.1 \mathrm{mHz}$. 
Figure 8 - Nyquist plot for conventional and interdigitated geometries under adiabatic condition.

The Nyquist plots are composed by semicircles (overall resistance) at higher and medium frequency and by a straight line at lower frequencies [45] as illustrated in figure 8 . Figure 8 shows that the conventional geometry shows higher internal overall resistance when compared to the interdigitated geometry. The internal overall resistance is $1.10 \times 10^{-4}$ $\Omega . \mathrm{m}^{2}$ and $5.94 \times 10^{-5} \Omega . \mathrm{m}^{2}$ for conventional and interdigitated geometries, respectively. Thus, considering the thermal model, the battery performance is a balance between the higher discharge capacity value caused by the increase of the temperature and the losses related to the internal overall resistance.

\subsubsection{Environmental conditions}

All geometries were subjected to three thermal external conditions considering initial thermal equilibrium with the environmental, whose temperature is cold, $265.15 \mathrm{~K}$, figures 9a) and 9b); regular, $298.15 \mathrm{~K}$, figures 9c) and 9d); and hot, $316.15 \mathrm{~K}$, figures 9e) and 9f).

For each case, the heat produced during the discharge process is exchanged with the exterior and for each geometry, the discharge capacity, the total heat (irreversible, reversible and ohmic heat) and internal temperature were evaluated as a function of the scan rate.

Figures 9a) and b) show the discharge capacity value and temperature as a function of the scan rate between $1 \mathrm{i}_{\text {app }}$ at $250 \mathrm{i}_{\text {app }}$, respectively, for cold condition and all geometries. None of the batteries can operate at scan rates above $250 \mathrm{i}_{\text {app }}$, as the low temperature $(265.15 \mathrm{~K})$ severely limits the diffusion and the ionic conductivity of the electrolyte solution [11]. The battery performance for all geometries is identical to the one observed for isothermal and adiabatic condition but with lower discharge capacity values. The conventional geometry only operates up to a scan rate of $17 \mathrm{i}_{\mathrm{app}}$, for which the discharge capacity value is $454 \mathrm{Ah} \cdot \mathrm{m}^{-2}$. 
At $250 \mathrm{i}_{\text {app }}$ the discharge capacity values are 234 Ah.m ${ }^{-2}, 194$ Ah.m ${ }^{-2}, 158$ Ah.m ${ }^{-2}, 212$ Ah.m ${ }^{-2}, 280$ Ah.m ${ }^{-2}$ and 319 Ah.m m $^{-2}$ for horseshoe, spiral, antenna, ring, interdigitated and gear geometries, respectively. The interdigitated geometry shows the best performance under isothermal and adiabatic condition but for cold conditions the values for both geometries (interdigitated and gear geometries) are very close. However, the gear geometry shows slightly better performance than the interdigitated geometry as the gear geometry has smaller distance between current collectors and lower distances for ions to move until their intercalation.

Figure 9 - Delivered capacity (left) and final temperature (right) as a function of the scan rate for all geometries under cold (a and b), regular (c and d) and hot (e and f) conditions.

In relation to the temperature (figure $9 \mathrm{~b}$ ), the conventional geometry reaches higher temperatures in comparison to the other geometries due to its higher internal overall resistance. The temperature value observed for interdigitated and gear geometries is due to the lower exchange of heat with the exterior when compared to the other geometries, resulting in higher discharge capacity values. The contact area between the battery and the exterior for the interdigitated and gear geometries is lower, affecting therefore the heat transfer process.

Figures 9c) and d) show the discharge capacity and temperature values, respectively for all geometries as a function of the scan rate $\left(1 \mathrm{i}_{\text {app }}\right.$ to $\left.500 \mathrm{i}_{\text {app }}\right)$ for regular environmental condition $(298.15 \mathrm{~K})$.

For this thermal condition, the conventional geometry can operate up to $300 \mathrm{i}_{\text {app }}$ and all other geometries up to $500 \mathrm{i}_{\text {app. }}$. At $300 \mathrm{i}_{\text {app }}$, the conventional geometry has the lowest discharge capacity value (318 Ah.m ${ }^{-2}$ ) and interdigitated geometry has the highest discharge capacity value (371 Ah. $\left.\mathrm{m}^{-2}\right)$ in comparison to the other geometries.

As it was observed under adiabatic condition, the increases of the diffusion and conductivity value due to the increase of temperature value leads to an increase in battery discharge capacity. It is to notice that the horseshoe, spiral, antenna and ring geometries do not show a increase in battery performance once these geometries have elevated distances for ions and electrons to move during the discharge process.

Figure 9d shows that the conventional geometry presents highest temperature value once it absorbs the heat produced due its internal overall resistance value. For this thermal 
condition, it is also observed that interdigitated and gear geometries have higher temperature value in comparison to the horseshoe, spiral, antenna and ring geometries for the same reason that was observed for the cold condition.

Figures 9e) and f) show the discharge capacity and temperature values, respectively, for all geometries as a function of the scan rate ( $1 \mathrm{i}_{\text {app }}$ to $\left.500 \mathrm{i}_{\text {app }}\right)$ for hot condition $(316.15 \mathrm{~K})$. For this thermal condition, differences in discharge capacity value are just observed at scan rates above $400 \mathrm{i}_{\text {app }}$ for all geometries. For this temperature, the conventional geometry operates up to $500 \mathrm{i}_{\text {app. }}$. Further, the differences in the discharge capacity values are small in comparison to the isothermal, adiabatic, cold and environmental conditions for all geometries

As previously observed, the interdigitated and gear geometries show the best discharge values in comparison to the other geometries. Relatively to the temperature behavior (figure 9f), the conventional geometry has the higher temperature value for all scan rates due to the higher separator thickness. The temperature behavior for the other geometries (figure 9f) is the same as observed for adiabatic and environmental conditions.

It is important to notice that the conventional geometry reaches temperatures above 323 $\mathrm{K}$ for scan rates above $300 \mathrm{i}_{\text {app }}$ where the organic solvent of the electrolyte solution can start to evaporate [46].

\subsection{Total heat at low and high discharge rates}

The total dissipated heat for the different geometries was evaluated with the objective to relate the increases of the temperature with the total heat produced by the battery.

The generated heat in the battery comes from three contributions: reaction, reversible and ohmic. The total heat of the different components (anode, separator and cathode) was determined for all geometries under adiabatic conditions at low scan rate $\left(1 i_{\text {app }}\right)$ and high scan rate $\left(300 \mathrm{i}_{\mathrm{app}}\right)$ once the conventional geometry only operates up to this scan rate.

Figure 10a), b) and c) show the total heat in the anode, separator and cathode, respectively, for all geometries at $1 \mathrm{i}_{\text {app }}$ as a function of time.

Figure 10 - Total heat in the anode (a), separator (b) and cathode (c) for all geometries at $1 i_{\text {app }}$ as a function of the time. d) Total heat along the battery for all geometries at $1 i_{\text {app }}$ after 120 000s. 
The total discharge time for all geometries at $1 i_{\text {app }}$ is around 150000 s. Figure $10 \mathrm{~d}$ ) shows the total heat for all geometries at different places between current collectors at time of 120000 s

Figure 10a) and c) show that the total heat produced by the electrodes (anode and cathode, respectively) is the same at all instants of time along the discharge cycle.

For all geometries, the total dissipated heat for anode changes between $-220 \mathrm{~W} / \mathrm{m}^{3}$ to 370 $\mathrm{W} / \mathrm{m}^{3}$ and for cathode varies between $0 \mathrm{~W} / \mathrm{m}^{3}$ to $110 \mathrm{~W} / \mathrm{m}^{3}$, as a function of time All geometries produce the same amount of heat in each electrode, being therefore identical the effect of losses associated with the internal resistance caused by the diffusion and conductivity of the ions and the electrical conduction.

The heat produced is the same in all geometries as at low discharge rates, a low ionic mobility is required and the internal resistance has not significant effects in the produced heat.

For the separator, figure $10 \mathrm{~b}$ ) shows that the total dissipated heat for conventional geometry is higher in comparison to the other geometries. Thus, for the conventional geometry the total dissipated heat varies from $8.8 \mathrm{~W} / \mathrm{m}^{3}$ to $9.5 \mathrm{~W} / \mathrm{m}^{3}$ as a function of time and for the other geometries the total dissipated heat varies between $0.01 \mathrm{~W} / \mathrm{m}^{3}$ to 0.56 $\mathrm{W} / \mathrm{m}^{3}$.

This fact is due to the higher separator thickness for the conventional geometry in comparison to the other geometries that affects the mobility of the ions and in turn the produced heat.

Figure $10 \mathrm{~d}$ ) shows the total heat at a time of $120000 \mathrm{~s}$ in different points between current collectors for all geometries, where the heat is produced according the results of the figure 10a) to c).

As represented in figure $10 \mathrm{~d}$ ) at $1 \mathrm{i}_{\text {app }}$, the total heat of the electrodes is very close for all geometries, the difference being verified for the separator due to their thicknesses.

Further, the evolution of the temperature of the battery as a function of time for all geometries is shown in figure 11 at $1 \mathrm{i}_{\text {app }}$.

Figure 11 - Temperature of the battery as a function of time for all geometries at $1 i_{\text {app }}$.

Figure 11 shows that the temperature of the batteries along the discharge time is independent of the geometry. 
Figures 12a) b) and c) show the total dissipated heat as a function of time in the anode, separator and cathode, respectively, for all geometries at $300 \mathrm{i}_{\text {app. }}$. This condition is selected at the higher scan rate will be produce a larger effect of the internal resistance during to the discharge process.

Figure 12a) shows that the geometries that produce a lower amount of heat in the anode are the gear and the interdigitated geometries.

In contrast, the conventional, ring, spiral and antenna geometries produce larger amounts of heat along to the discharge time.

Figure 12 - Total heat for anode (a), separator (b) and cathode (c) for all geometries at $300 \mathrm{i}_{\text {app }}$ as a function of time.

It is to notice that the antenna, ring and spiral geometries are characterized by small distances between current collectors and also small separator thickness as well as longer distances for the ions to move (d_max). This fact implies higher dissipated heat due to ohmic losses. It is interesting to notice that close to the end of the discharge time, the ring, antenna, spiral and horseshoe geometries approach to the behavior of the conventional geometry. In this case, the total dissipated heat for these geometries increases over time due to the contribution of the ions located in places more distant from the collectors.

Identical behavior is observed for the cathode (figure 12c), where the conventional geometry shows higher total dissipated heat $\left(270 \mathrm{~kW} / \mathrm{m}^{3}\right)$ in comparison to the other geometries and the geometry with the lower total dissipated heat is the gear geometry (50 $\left.\mathrm{kW} / \mathrm{m}^{3}\right)$.

Relatively to the separator (figure 12b), the conventional geometry also shows higher total dissipated heat in comparison to the other geometries, with values between 388 $\mathrm{kW} / \mathrm{m}^{3}$ and $810 \mathrm{~kW} / \mathrm{m}^{3}$. The interdigitated and gear geometries show intermediate values of $39 \mathrm{~kW} / \mathrm{m}^{3}$ and $14 \mathrm{~kW} / \mathrm{m}^{3}$, whereas the dissipate heat is between $1071 \mathrm{~W} / \mathrm{m}^{3}$ to $7200 \mathrm{~W} / \mathrm{m}^{3}$ for the other geometries. In this case, the conventional geometry shows higher total dissipated heat due to the higher separator thickness in comparison to the other geometries (table 3) and the interdigitated and gear geometries show intermediate values due to the separator thickness and distance between current collectors.

Figures 13 a) and b) show the total heat in different positions on the battery between the current collectors for all geometries after $50 \mathrm{~s}$ at $300 \mathrm{i}_{\text {app }}$. 
Figure 13 - Total heat along the battery after $50 \mathrm{~s}$ at $300 \mathrm{i}_{\text {app }}$ for conventional and interdigitated geometries (a) and for the remaining geometries (b).

Figure 13a) shows that batteries with conventional geometry generate higher heat due to the thickness of the separator. Figure 13 also shows that the interdigitated and gear geometries generate lower heat values in all positions between the current collectors due to the lower thickness of separator.

The geometries that produce lower total dissipated heat are the interdigitated and gear geometries, which is related to the thickness of the separator and distance between current collectors.

Figure 14 shows the evolution of the temperature of the battery as a function of time with the different geometries at $300 \mathrm{i}_{\text {app }}$.

Figure 14 - Temperature as a function of time for all geometries at $300 \mathrm{i}_{\text {app }}$.

Figure 14 shows that the temperature of the different batteries increases linearly in time. The conventional geometry temperature increases from $298.15 \mathrm{~K}$ to $318.10 \mathrm{~K}$, whereas for the interdigitated and gear geometries the increase is the lowest reaching a maximum of $310 \mathrm{~K}$ after $260 \mathrm{~s}$ discharge time.

\subsection{Ohmic heat for ring geometry with different radius}

For understanding the contribution of the mobility of the ions and the corresponding resistance to produce heat and its influence into battery performance, this section analyzes the ohmic heat produced in a ring geometry for different radius at $500 \mathrm{i}_{\text {app }}$ and under adiabatic conditions. The selected radius are $93.9 \mu \mathrm{m}, 230 \mu \mathrm{m}, 330 \mu \mathrm{m}$ and $430 \mu \mathrm{m}$ (Figure 15), the ohmic heat being associated to the Joule effect caused by the ohmic losses. The ohmic losses are due to the different paths the charges have to move to the current collectors (d_max) and also to differences in the thickness of the separator. The variation of the radius is carried out while maintaining constant the area of all components of battery (electrodes, separator and current collectors). 
Figure 15 - Schematic representation of the ring geometry for the radius of $93.9 \mu \mathrm{m}$ and $430 \mu \mathrm{m}$.

Simulations show (Figure 16 a)) that the capacity of the battery decreases with increasing the radius of the ring. The ring geometry with bigger radius has smaller thickness for electrodes and separator, lower distance between the current collector, and higher maximum distance for the ions (d_max) that implies lower battery performance due to the higher internal resistance.

It is observed in figure $16 \mathrm{~b}$ ) that the temperature of the battery increases over time for all radii, being the increase larger for the larger radius.

Figure 16-a) Capacity as a function of ring radius and b) temperature as a function of time for all ring radius at $500 \mathrm{i}_{\text {app }}$.

The heat for each component (electrodes and separator) as a function of time for the batteries with the different radius is shown in figure 17.

Figure 17 a) and c) show that for both electrodes (anode and cathode) the generated heat increase with increasing radius.

As previously indicated, higher ohmic losses are observed for the geometry with the larger radius due to the increase of the path the charges must move during the discharge process.

Figure 17 - Ohmic heat for anode (a), separator (b) and cathode (c) as a function of the time at $500 \mathrm{i}_{\text {app }}$ for various ring radius.

Figure $17 \mathrm{~b}$ ) shows the ohmic heat at the separator for the ring geometries with different radius. Contrary to the observations for the electrodes (figure 17a) and c)), the higher ohmic heat is observed for the battery with the smaller radius. In this case, the ring geometry with smaller radius produces higher ohmic heat due to the higher separator thickness. This fact will affect the diffusion and conduction behavior of the ions and consequently will increase by the Joule effect, leading to higher ohmic heat.

Figure 18 shows the ohmic heat fin the battery along different positions between the current collectors for the ring geometry with different radius at $70 \mathrm{~s}$ and $500 \mathrm{i}_{\text {app. }}$. 
Figure 18 - Ohmic heat along different places between the current collectors of the battery after $70 \mathrm{~s}$ at $500 \mathrm{i}_{\text {app }}$ for ring geometry with different radius.

Considering figure 18, it is observed higher ohmic heat for the electrodes of the ring geometry with higher radius due to the larger distance the ions have to move until intercalation.

In relation to the separator, the higher ohmic heat is observed for the smaller radius due to higher separator thickness.

Figure 19 shows the impedance curves for the ring geometry with different radii in order to determine the internal resistance value.

Independently of the radius of the ring geometry, the Nyquist plot is characterized by two semicircles at high frequencies identified in the figure 19 where the overall resistance that is the sum of the two semicircles that represent the ohmic resistance, which is related to the contact film resistance and resistance contributions from the charge-transfer reaction resistance in the high and medium frequency range. At low-frequency range, the inclined line corresponds to the Warburg impedance, associated to the lithium-ion diffusion in the bulk of the active material [47]. The diameter of the semicircles corresponds to the total impedance and its value is $5 \times 10^{-5} \Omega . \mathrm{m}^{2}, 6 \times 10^{-5} \Omega . \mathrm{m}^{2}, 8 \times 10^{-5} \Omega . \mathrm{m}^{2}$ and $9 \times 10^{-5} \Omega . \mathrm{m}^{2}$ for $93.9 \mu \mathrm{m}, 230 \mu \mathrm{m}, 330 \mu \mathrm{m}$ and $430 \mu \mathrm{m}$, respectively.

Figure 19 - Nyquist plot for the ring geometry with different radius at $500 \mathrm{i}_{\text {app }}$.

It is observed that the ring geometry with small radius shows the lowest resistance value due to the smaller paths that the ions placed distant from current collectors must move. To understand the effect of the maximum distance of the ions until the intercalation process, figure 20 shows the ionic current density vectors for the ring geometry. Figures 20a) and b) show the ionic current density vectors for the batteries with the smaller and higher radius, respectively that correspond to the ionic charge at the time of $70 \mathrm{~s}$ at $500 \mathrm{i}_{\text {app. }}$. This magnitude is represented as a vector on the 2D graphics shown in figure 20, indicating the direction of the ions. 
Figure 20 - Ionic current density vectors of the ring geometry for a) $\mathrm{R}=93.9 \mu \mathrm{m}$ and $\mathrm{b}$ ) $\mathrm{R}=430 \mu \mathrm{m}$

Figure 20 a) shows that for the battery with the smaller radius, the most distant ions have a shorter maximum distance to travel to the current collectors. In relation of the battery with bigger radius (figure 20b), it is observed a similar behavior. Figure 20b) also shows that for places closer to the current collectors, the ionic current density is more intense, the ring geometry with higher radius dissipating more ohmic heat.

It is concluded that battery performance for each geometry can be optimized considering the geometrical parameters that will be influence the thermal behavior.

\section{Conclusions}

Thermal properties are a critical issue in lithium-ion batteries as they strongly influence battery performance and safety. Thus, for maximizing battery performance it is essential to properly analyze the effect of the thermal behavior of different battery geometries through computer simulations. This work shows the effect of the thermal conditions (isothermal, adiabatic, cold, regular and hot conditions) in different battery geometries, including conventional (layer by layer) and unconventional geometries (interdigitated, horseshoe, spiral, ring, antenna and gear geometries). The simulations were based on the Newman/Doyle/Fuller model with a coupled thermal model. Under isothermal and adiabatic conditions, the best geometries are interdigitated and gear geometries due to higher battery performance and low temperature values relatively to the other geometries and the main reason for this behavior is the smaller separator thickness, lower distances for the ions to move (d_max) and higher contact surface area of the electrodes.

The best battery performance is obtained for the gear geometry at cold condition (265.15 $\mathrm{K})$. For regular and hot thermal conditions the best geometries are the gear and interdigitated ones. The generated heat mostly depends on the internal resistance related to the maximum distances that ions move until its intercalation and on the thickness of the separator.

Thus, this work demonstrates how the battery performance can be optimized for specific geometries considering different thermal conditions. The results shown in this work are an essential step for the applicability of these geometries into specific applications, once 
they can be fabricated by 3D-printing techniques. Further, considering that the interdigitated geometry presents excellent cycling properties, it will be further interesting to evaluate the effect of the thermal properties considering different active materials for the cathode.

\section{Acknowledgments}

This work was supported by the Portuguese Foundation for Science and Technology (FCT) in the framework of the Strategic Funding UID/FIS/04650/2013. The authors thank FEDER funds through the COMPETE 2020 Programme and National Funds through FCT under the projects PTDC/CTM-ENE/5387/2014 and UID/CTM/50025/2013 and grant SFRH/BPD/112547/2015 (C.M.C.). Financial support from the Basque Government Industry Department under the ELKARTEK Program is also acknowledged.

\section{Nomenclature}

\begin{tabular}{|c|c|}
\hline \multicolumn{2}{|c|}{ List of symbols } \\
\hline $\mathrm{a}$ & specific interfacial area, $\mathrm{m}^{2} / \mathrm{m}^{3}$ \\
\hline $\mathrm{C}_{\mathrm{L}}$ & concentration of $\mathrm{Li}$ ions in the electrolyte, $\mathrm{mol} / \mathrm{m}^{3}$ \\
\hline $\mathrm{C}_{\mathrm{E}}$ & concentration of $\mathrm{Li}$ ions in the electrode, $\mathrm{mol} / \mathrm{m}^{3}$ \\
\hline $\mathrm{D}_{\mathrm{i}}(\mathrm{c}, \mathrm{T})$ & $\begin{array}{l}\text { diffusion coefficient of the salt in the electrolyte as a function of lithium } \\
\text { concentration and temperature } i(i=\mathrm{a}, \mathrm{s}, \mathrm{c}), \mathrm{m}^{2} / \mathrm{s}\end{array}$ \\
\hline $\mathrm{D}_{\mathrm{ef}, \mathrm{i}}$ & effective diffusion coefficient of the salt in the electrolyte $i(i=\mathrm{a}, \mathrm{s}, \mathrm{c}), \mathrm{m}^{2} / \mathrm{s}$ \\
\hline $\mathrm{D}_{\mathrm{LI}, \mathrm{i}}(\mathrm{T})$ & $\begin{array}{l}\text { diffusion coefficient of } \mathrm{Li} \text { ions in the electrode as a function of temperature, } \\
\mathrm{m}^{2} / \mathrm{s}\end{array}$ \\
\hline $\mathrm{D}_{\mathrm{LI}, 298.15 \mathrm{~K}}$ & diffusion coefficient of $\mathrm{Li}$ ions in the electrode at $298.15 \mathrm{~K} i(i=\mathrm{a}, \mathrm{c}), \mathrm{m}^{2} / \mathrm{s}$ \\
\hline $\mathrm{F}$ & Faraday's constant, $96487 \mathrm{C} / \mathrm{mol}$ \\
\hline $\mathrm{i}_{\text {app }}$ & applied current density, $\mathrm{A} / \mathrm{m}^{2}$ \\
\hline $\mathbf{i}_{\mathrm{E}}$ & current density in the electrode, $\mathrm{A} / \mathrm{m}^{2}$ \\
\hline $\mathbf{i}_{\mathrm{L}}$ & current density in the electrolyte phase, $\mathrm{A} / \mathrm{m}^{2}$ \\
\hline $\mathrm{I}_{\text {TOTAL }}$ & total current density, $\mathrm{A} / \mathrm{m}^{2}$ \\
\hline $\mathrm{j}_{\mathrm{Li}}^{+}$ & pore wall flux of $\mathrm{Li}$ ions, $\mathrm{mol} / \mathrm{cm}^{2} \mathrm{~s}$ \\
\hline$K_{t, i}(T)$ & $\begin{array}{l}\text { reaction rate coefficient of the electrodes as a function of temperature } i(i=\mathrm{a}, \mathrm{c}) \text {, } \\
\mathrm{m} / \mathrm{s}\end{array}$ \\
\hline$K_{t 298,15, i}$ & reaction rate coefficient of the electrodes at $298.15 \mathrm{~K} i(i=\mathrm{a}, \mathrm{c}), \mathrm{m} / \mathrm{s}$ \\
\hline L_i & width of the electrode $i(i=\mathrm{a}, \mathrm{c}), \mathrm{m}$ \\
\hline L_dim & dimension of the horseshoe geometry, $\mathrm{m}$ \\
\hline
\end{tabular}


cdig

edig

e_sep

d_max

d_cc

$\mathrm{R}_{\mathrm{d}}$

$\mathrm{R}_{\mathrm{g}}$

M

$\mathbf{R}$

$\mathrm{R}$

$\mathrm{R}_{\mathrm{f}}$

r

$\mathrm{T}$

$\mathrm{T}_{\infty}$

$\mathrm{t}$

$t_{+}^{0}$

$u^{0}$

$\mathrm{N}$

$\mathrm{A}_{\mathrm{i}}$

brugg

$E_{a d, i}$

$E_{a k, i}$

$\mathrm{C}_{\mathrm{p}, \mathrm{i}}$

$\mathrm{h}$

$\mathrm{Q}_{\text {total,i }}$

Qohmic,i

Qreversible

$\mathrm{Q}_{\text {reaction, } \mathrm{i}}$

digit length of the electrode, $\mathrm{m}$

digit thickness of the electrode, $\mathrm{m}$

separator thickness, $\mathrm{m}$

maximum distance of ions more distant of collectors positions

distance between of collectors

radius of ring geometry, $\mathrm{m}$

radius of gear geometry, $\mathrm{m}$

mass transport flux, $\mathrm{mol} / \mathrm{m}^{2}$

reaction term of the mass balance equation, $\mathrm{mol} / \mathrm{m}^{3} \mathrm{~s}$

gas constant, 8,314 J/mol K

film resistance, $\Omega \mathrm{m}^{2}$

radius of the electrode spherical particles, $\mathrm{m}$

temperature, $\mathrm{K}$

external temperature, $\mathrm{K}$

time, $s$

transport number of the positive ions

open circuit voltage, $\mathrm{V}$

number of digits for interdigitated and gear battery

area of a given component in the battery $i(i=\mathrm{a}, \mathrm{s}, \mathrm{c})$

brugg parameter in the electrodes and separator

activation energy for diffusion of electrodes $i(i=\mathrm{a}, \mathrm{c}), \mathrm{J} / \mathrm{mol}$

activation energy for reation of electrodes $i(i=\mathrm{a}, \mathrm{c}), \mathrm{J} / \mathrm{mol}$

heat capacity at constant pressure of battery components $i(i=\mathrm{a}, \mathrm{s}, \mathrm{c}), \mathrm{J} /(\mathrm{kg} . \mathrm{K})$

heat transfer coefficient, $\mathrm{W} /\left(\mathrm{m}^{2} . \mathrm{K}\right)$

total heat generation rate of battery components $i(i=\mathrm{a}, \mathrm{s}, \mathrm{c}), \mathrm{W} / \mathrm{m}^{3}$

total ohmic heat generation rate of battery components $i(i=\mathrm{a}, \mathrm{s}, \mathrm{c}), \mathrm{W} / \mathrm{m}^{3}$

total reversible heat generation rate of electrodes $i(i=\mathrm{a}, \mathrm{c}), \mathrm{W} / \mathrm{m}^{3}$

total reaction heat generation rate of electrodes $i(i=\mathrm{a}, \mathrm{c}), \mathrm{W} / \mathrm{m}^{3}$

\section{Greek symbols}

$\varepsilon_{\mathrm{i}} \quad$ porosity of region $i(i=\mathrm{a}, \mathrm{s}, \mathrm{c})$

$\varepsilon_{\mathrm{f}, \mathrm{i}} \quad$ volume fraction of the fillers in the electrode $i(i=\mathrm{a}, \mathrm{s}, \mathrm{c})$

$\tau \quad$ tortuosity of the separator

$\eta \quad$ over-potential, $\mathrm{V}$

$\varphi_{E} \quad$ potential of the electrodes, $\mathrm{V}$ 


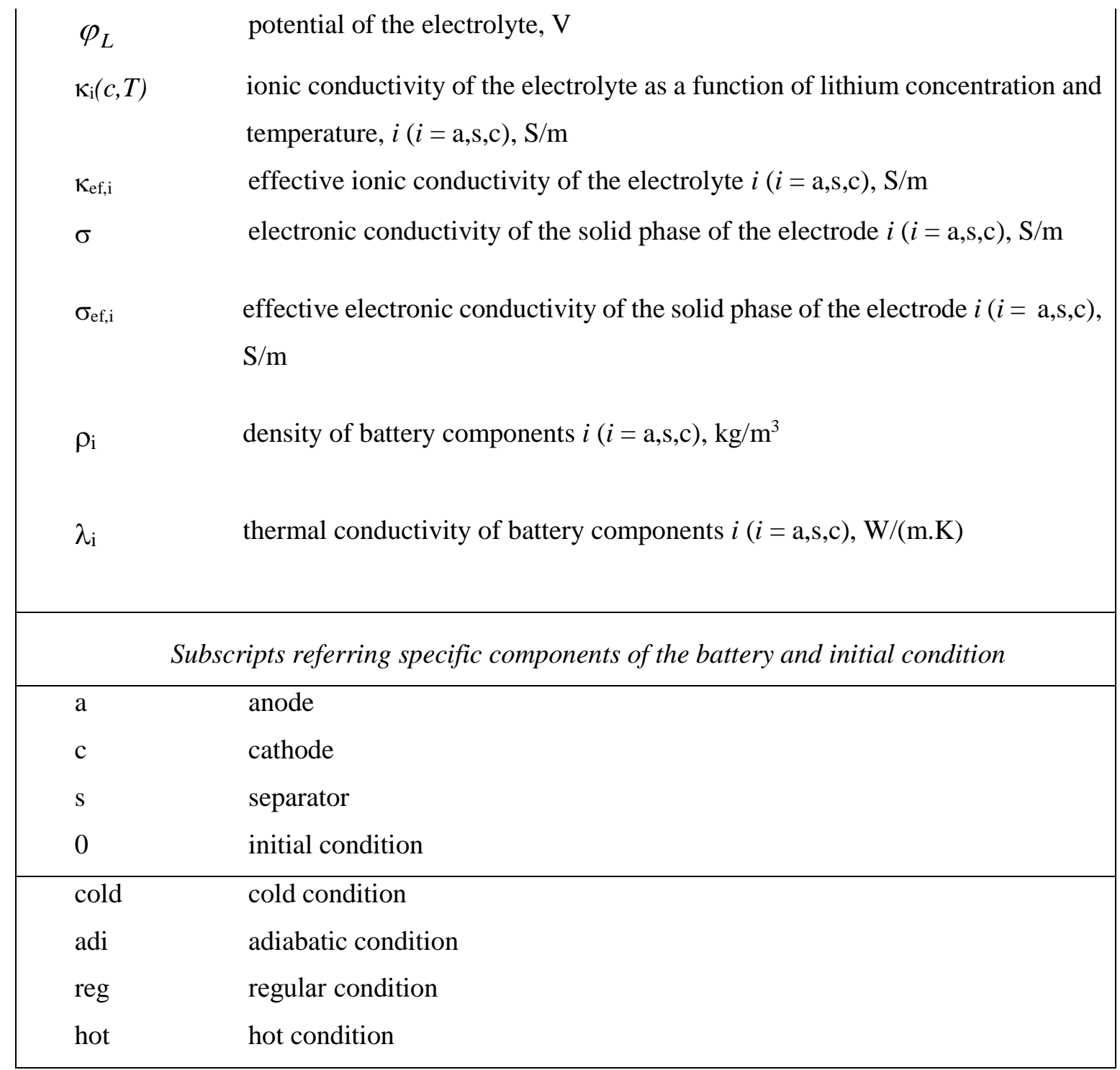




\section{References}

[1] Dunn B, Kamath H, Tarascon J-M. Electrical Energy Storage for the Grid: A Battery of Choices. Science. 2011;334:928-35.

[2] Lin X, Salari M, Arava LMR, Ajayan PM, Grinstaff MW. High temperature electrical energy storage: advances, challenges, and frontiers. Chemical Society Reviews. 2016;45:5848-87.

[3] Hu X, Martinez CM, Yang Y. Charging, power management, and battery degradation mitigation in plug-in hybrid electric vehicles: A unified cost-optimal approach. Mechanical Systems and Signal Processing. 2017;87, Part B:4-16.

[4] Wu X, Hu X, Moura S, Yin X, Pickert V. Stochastic control of smart home energy management with plug-in electric vehicle battery energy storage and photovoltaic array. Journal of Power Sources. 2016;333:203-12.

[5] Ozawa K. Lithium Ion Rechargeable Batteries: Materials, Technology, and New Applications: Wiley; 2012.

[6] Tarascon J-M. Key challenges in future Li-battery research. Philosophical Transactions of the Royal Society A: Mathematical,

Physical and Engineering Sciences. 2010;368:3227-41.

[7] Scrosati B, Garche J. Lithium batteries: Status, prospects and future. Journal of Power Sources. 2010;195:2419-30.

[8] Khateeb SA, Amiruddin S, Farid M, Selman JR, Al-Hallaj S. Thermal management of Li-ion battery with phase change material for electric scooters: experimental validation. Journal of Power Sources. 2005;142:345-53.

[9] Balakrishnan PG, Ramesh R, Prem Kumar T. Safety mechanisms in lithium-ion batteries. Journal of Power Sources. 2006;155:401-14.

[10] Wang Q, Ping P, Zhao X, Chu G, Sun J, Chen C. Thermal runaway caused fire and explosion of lithium ion battery. Journal of Power Sources. 2012;208:210-24.

[11] Park M, Zhang X, Chung M, Less GB, Sastry AM. A review of conduction phenomena in Li-ion batteries. Journal of Power Sources. 2010;195:7904-29.

[12] Mills A, Al-Hallaj S. Simulation of passive thermal management system for lithiumion battery packs. Journal of Power Sources. 2005;141:307-15. 
[13] Sabbah R, Kizilel R, Selman JR, Al-Hallaj S. Active (air-cooled) vs. passive (phase change material) thermal management of high power lithium-ion packs: Limitation of temperature rise and uniformity of temperature distribution. Journal of Power Sources. 2008;182:630-8.

[14] Pesaran AA. Battery thermal management in EV and HEVs: issues and solutions. Battery Man. 2001;43:34-49.

[15] Rao Z, Wang S, Wu M, Lin Z, Li F. Experimental investigation on thermal management of electric vehicle battery with heat pipe. Energy Conversion and Management. 2013;65:92-7.

[16] Chen K, Song M, Wei W, Wang S. Structure optimization of parallel air-cooled battery thermal management system with U-type flow for cooling efficiency improvement. Energy. 2018;145:603-13.

[17] Ardani MI, Patel Y, Siddiq A, Offer GJ, Martinez-Botas RF. Combined experimental and numerical evaluation of the differences between convective and conductive thermal control on the performance of a lithium ion cell. Energy. 2018;144:81-97.

[18] Samimi F, Babapoor A, Azizi M, Karimi G. Thermal management analysis of a Liion battery cell using phase change material loaded with carbon fibers. Energy. 2016;96:355-71.

[19] Situ W, Zhang G, Li X, Yang X, Wei C, Rao M, et al. A thermal management system for rectangular LiFePO4 battery module using novel double copper mesh-enhanced phase change material plates. Energy. 2017.

[20] Marzband M, Moghaddam MM, Akorede MF, Khomeyrani G. Adaptive load shedding scheme for frequency stability enhancement in microgrids. Electric Power Systems Research. 2016;140:78-86.

[21] Marzband M, Yousefnejad E, Sumper A, Domínguez-García JL. Real time experimental implementation of optimum energy management system in standalone Microgrid by using multi-layer ant colony optimization. International Journal of Electrical Power \& Energy Systems. 2016;75:265-74.

[22] Marzband M, Ghadimi M, Sumper A, Domínguez-García JL. Experimental validation of a real-time energy management system using multi-period gravitational search algorithm for microgrids in islanded mode. Applied Energy. 2014;128:164-74.

[23] Marzband M, Sumper A, Ruiz-Álvarez A, Domínguez-García JL, Tomoiagă B. Experimental evaluation of a real time energy management system for stand-alone microgrids in day-ahead markets. Applied Energy. 2013;106:365-76. 
[24] Wang T, Tseng KJ, Zhao J, Wei Z. Thermal investigation of lithium-ion battery module with different cell arrangement structures and forced air-cooling strategies. Applied Energy. 2014;134:229-38.

[25] Eddahech A, Briat O, Vinassa J-M. Thermal characterization of a high-power lithium-ion battery: Potentiometric and calorimetric measurement of entropy changes. Energy. 2013;61:432-9.

[26] Zhao R, Zhang S, Liu J, Gu J. A review of thermal performance improving methods of lithium ion battery: Electrode modification and thermal management system. Journal of Power Sources. 2015;299:557-77.

[27] Zhao R, Liu J, Gu J. The effects of electrode thickness on the electrochemical and thermal characteristics of lithium ion battery. Applied Energy. 2015;139:220-9.

[28] Du W, Gupta A, Zhang X, Sastry AM, Shyy W. Effect of cycling rate, particle size and transport properties on lithium-ion cathode performance. International Journal of Heat and Mass Transfer. 2010;53:3552-61.

[29] Miranda D, Costa CM, Almeida AM, Lanceros-Méndez S. Computer simulations of the influence of geometry in the performance of conventional and unconventional lithium-ion batteries. Applied Energy. 2016;165:318-28.

[30] Dong T, Peng P, Jiang F. Numerical modeling and analysis of the thermal behavior of NCM lithium-ion batteries subjected to very high C-rate discharge/charge operations. International Journal of Heat and Mass Transfer. 2018;117:261-72.

[31] Yeow K, Teng H, Thelliez M, Tan E. 3D thermal analysis of Li-ion battery cells with various geometries and cooling conditions using Abaqus. Proceedings of the SIMULIA Community Conference2012.

[32] Cai L, White RE. Mathematical modeling of a lithium ion battery with thermal effects in COMSOL Inc. Multiphysics (MP) software. Journal of Power Sources. 2011;196:5985-9.

[33] Feng X, Lu L, Ouyang M, Li J, He X. A 3D thermal runaway propagation model for a large format lithium ion battery module. Energy. 2016;115:194-208.

[34] Zheng S, Wang L, Feng X, He X. Probing the heat sources during thermal runaway process by thermal analysis of different battery chemistries. Journal of Power Sources. 2018;378:527-36.

[35] Gören A, Mendes J, Rodrigues HM, Sousa RE, Oliveira J, Hilliou L, et al. High performance screen-printed electrodes prepared by a green solvent approach for lithiumion batteries. Journal of Power Sources. 2016;334:65-77. 
[36] Srinivasan V, Newman J. Discharge Model for the Lithium Iron-Phosphate Electrode. Journal of The Electrochemical Society. 2004;151:A1517-A29.

[37] Northrop PWC, Pathak M, Rife D, De S, Santhanagopalan S, Subramanian VR. Efficient Simulation and Model Reformulation of Two-Dimensional Electrochemical Thermal Behavior of Lithium-Ion Batteries. Journal of The Electrochemical Society. 2015;162:A940-A51.

[38] Gerver RE, Meyers JP. Three-Dimensional Modeling of Electrochemical Performance and Heat Generation of Lithium-Ion Batteries in Tabbed Planar Configurations. Journal of The Electrochemical Society. 2011;158:A835-A43.

[39] Bae S, Song HD, Nam I, Kim G-P, Lee JM, Yi J. Quantitative performance analysis of graphite-LiFePO4 battery working at low temperature. Chemical Engineering Science. 2014;118:74-82.

[40] Miranda D, Costa CM, Almeida AM, Lanceros-Méndez S. Computer simulation evaluation of the geometrical parameters affecting the performance of two dimensional interdigitated batteries. Journal of Electroanalytical Chemistry. 2016;780:1-11.

[41] Wang C, Hong J. Ionic/Electronic Conducting Characteristics of LiFePO4 Cathode Materials: The Determining Factors for High Rate Performance. Electrochemical and Solid-State Letters. 2007;10:A65-A9.

[42] Shetzline JA, Creager SE. Quantifying Electronic and Ionic Conductivity Contributions in Carbon/Polyelectrolyte Composite Thin Films. Journal of The Electrochemical Society. 2014;161:H917-H23.

[43] Prada E, Di Domenico D, Creff Y, Bernard J, Sauvant-Moynot V, Huet F. Simplified Electrochemical and Thermal Model of LiFePO4-Graphite Li-Ion Batteries for Fast Charge Applications. Journal of The Electrochemical Society. 2012;159:A1508-A19.

[44] Okamoto E, Nakamura M, Akasaka Y, Inoue Y, Abe Y, Chinzei T, et al. Analysis of Heat Generation of Lithium Ion Rechargeable Batteries Used in Implantable Battery Systems for Driving Undulation Pump Ventricular Assist Device. Artificial Organs. 2007;31:538-41.

[45] Solchenbach S, Pritzl D, Kong EJY, Landesfeind J, Gasteiger HA. A Gold MicroReference Electrode for Impedance and Potential Measurements in Lithium Ion Batteries. Journal of The Electrochemical Society. 2016;163:A2265-A72.

[46] Aurbach D, Talyosef Y, Markovsky B, Markevich E, Zinigrad E, Asraf L, et al. Design of electrolyte solutions for Li and Li-ion batteries: a review. Electrochimica Acta. 2004;50:247-54. 
[47] Zhu JG, Sun ZC, Wei XZ, Dai HF. A new electrochemical impedance spectroscopy model of a high-power lithium-ion battery. RSC Advances. 2014;4:29988-98. 